Article

\title{
Achieving Neuroplasticity in Artificial Neural Networks through Smart Cities
}

\author{
Zaheer Allam $\mathbb{D}$ \\ Curtin University Sustainability Policy Institute, Curtin University, Perth, WA 6102, Australia; \\ zaheerallam@gmail.com
}

Received: 12 February 2019; Accepted: 2 April 2019; Published: 8 April 2019

\begin{abstract}
Through the Internet of things (IoT), as promoted by smart cities, there is an emergence of big data accentuating the use of artificial intelligence through various components of urban planning, management, and design. One such system is that of artificial neural networks (ANNs), a component of machine learning that boasts similitude with brain neurological networks and its functioning. However, the development of ANN was done in singular fashion, whereby processes are rendered in sequence in a unidimensional perspective, contrasting with the functions of the brain to which ANN boasts similitude, and in particular to the concept of neuroplasticity which encourages unique complex interactions in self-learning fashion, thereby encouraging more inclusive urban processes and render urban coherence. This paper takes inspiration from Christopher Alexander's Nature of Order and dwells in the concept of complexity theory; it also proposes a theoretical model of how ANN can be rendered with the same plastic properties as brain neurological networks with multidimensional interactivity in the context of smart cities through the use of big data and its emerging complex networks. By doing so, this model caters to the creation of stronger, richer, and more complex patterns that support Alexander's concept of "wholeness" through the connection of overlapping networks. This paper is aimed toward engineers with interdisciplinary interest looking at creating more complex and intricate ANN models, and toward urban planners and urban theorists working on the emerging contemporary concept of smart cities.
\end{abstract}

Keywords: artificial intelligence; smart cities; artificial neural networks (ANNs); neuroplasticity; complexity; geometry; brain; machine learning

\section{Introduction}

Cities all over the world are confronted with the challenge of rapid urbanization and population increase, with over two-thirds of the population projected to be living in cities by 2050 [1]. These challenges prompted an increase in other urban issues like social inequalities, pollution, unequal housing, slow economic growth, and environmental degradation, among many others [2]. In addition to the numerous solutions that were tried and tested to overcome these issues, the concept of smart cities is taunted as one of the most potent [3,4], and its adoption in cities is gaining traction [5-7]. Its ability to allow for the integration and inter-linkage of different elements of a city's fabric yielded momentous results in areas where it was implemented; hence, most cities, both in developed and developing economies, were seen to embrace its adoption [8]. One of the salient features of the smart city concept is its reliance on technologies such as Internet of things (IoT) [9,10], big data [11,12], blockchain [13-15], and artificial intelligence (AI) [16,17], which transformed the design, planning, and management of urban life. These technologies allowed for speedy, quality, efficient, and real-time processes due to their ability to allow for massive data gathering and analysis [18,19]. They allowed communication between different components of the city within the network; hence, services and 
activities run seamlessly with little negative impact on the environment, the economy, and the available resources [20].

Another technological concept that is gaining traction is that of artificial neural networks (ANNs), a form of machine learning that has a wide range of applications, which, when employed in cities, can enhance the understanding of the urban fabric. For instance, Mathaini, et al. [21] used ANN to model future urban growth probability in India due to its ability to capture non-linear and immaterial complex behavior of the urban fabric. A similar study by Mathaini [22] simulated the urban growth in India and revealed that ANN could be used alongside other spatial techniques like geographic information systems (GIS) and remote sensing. Yeh and Li [23] highlighted that ANN allows for superior levels of performance and results compared to other universally known statistical models because of its ability to manage intricate spatial data in determining urban land use. Sorda [24] explained that ANN is a form of machine learning that is able to automate and improve on data from IoT and AI and other technologies and produce accurate results. He argued that one of its most interesting features is its ability to mirror human brains by allowing for adaptive learning, self-organization, and fault tolerance features. With these qualities, ANN models can be made to learn and improve upon their performance and operation with minimal human programming while yielding high-quality results. These qualities render ANN as a better candidate for the smart city concept, especially due to the fact that results have minimal or no errors. This is of particular importance in order to improve the efficiency of smart cities, for which operations ought to be error-free to avoid system failures.

While the applications of ANN are hailed by scientists, majorly due to its quality of self-learning which is similar to neurological patterns of the human brain, there are limited applications to cities, where it is contended that there is much to be done on this front. However, there is a clear disparity and lack of multi-disciplinary approaches to study the application of ANN in cities in a clear coherent fashion, and even to study how the processes of ANN can be improved to maximize the benefits that urban dwellers derive from the smart city concept.

Literature points to the understanding that the customization of ANN was applied to urban fabric; however, unfortunately, this was done in isolation by computer scientists who are meticulous in their field, but who have limited understanding of the complex theories of both biological entities and urbanism. This means that, despite the numerous benefits that ANN promises in predicting different patterns and behavior of different urban elements, other factors like the effectiveness of those predictions through its geometrical interactions may not be well captured, thus impacting on urban management, governance structures, and decision-making. This gap can lead to the overlooking of important details emerging from the complex interactions of urban systems, thereby resulting in decontextualized solutions or conclusions divergent from the realities in place.

This implies that, due to the intricate and complex nature of urban systems, any program that is aimed at understanding the urban fabric cannot be developed in isolation without factoring in the complex matters that appertain to urbanism. This line of thought is supported in the engineering domain, where Dubey, et al. [25] affirmed that a program cannot be developed in isolation and be expected to be universally compatible with different aspects of given areas and larger city centers. However, they support that, even though different cities have specific characteristics, artificial neural network programs can be developed and applied with some input from crowd sourcing. Kropp [26] adopted a similar approach when utilizing data for urban analysis. Even though there was widespread adoption, the potential of this system, due to its machine learning capabilities, is promising for its numerous benefits that it can bring to the urban fabric. Zorins and Grabusts [27], however, underlined that ANN is still in its infancy for real problem-solving; hence, cooperation and input guided by theories and principles from different fields become paramount.

This paper, thus, explores how ANN processes can be improved in their application in the urban fabric through the use of emerging networks in smart cities. A theoretical model is proposed to 
provide enhanced gathering and processing of data garnered from different sensors of smart cities, thus providing improved user service delivery free from errors and inconveniences.

\section{Artificial Neural Networks}

There are varying definitions for ANNs, but there is notable convergence toward its origin and functioning. Graupe [28] defined ANNs as computational networks which attempt to simulate, in an overarching fashion, the networks of nerve cells of the biological central nervous system. A similar definition was given by Jain and Fanelli [29], who viewed ANNs as a new generation of information-processing paradigms designed to mimic some of the behaviors of the human brain. Mehrotra, et al. [30] opined that there is a clear conjuncture that ANNs, as computing systems, were inspired from the analogy of biological neural networks [31]. Even though such similarities were drawn, there is emerging literature that supports that the brains of mammals are so advanced, such that even the most advanced computers cannot equate to the processing power of the human brain [32]. Hence, computer scientists believe that, by studying behavioral and brain patterns of humans, as well as human responses and reactions, and by adapting and integrating them into computer systems, there will be an emergence of intelligence [33]. The intelligence factor is then seen to have the potential to increase the appropriateness for handling and processing complex tasks at high speed with minimal errors. ANNs are, thus, valued for their accuracy, speed, latency, volume, convergence, scalability, fault tolerance, and performance [34].

Like human brains, which have to learn and be trained to understand different things, artificial neural networks undergo an almost similar trend when developed. Human brains are made of countless cells, and their biological neural networks function via the interconnection of hundreds of trillions of synapses which serve as storage for information [32,35]. Noting the complexity of human brains, ANNs are nowhere near this quality in terms of processing power [27]. ANNs are believed to comprise hundreds of neurons which are insignificant relative to biological neurons. One notable difference between ANNs and the biological brain is that animals, especially man, have the ability to train and learn different activities simultaneously, while ANNs train for particular tasks one by one. Another difference, as explained by Perwej and Parwej [36], is that ANNs cannot emulate the neuroplasticity of a human brain; this is elaborated further in the next section of this paper. After training, the biological brain is said to be in a position to use previous representations of the problem to effectively respond to a new challenge or a stimulus which is totally new; with time, it is then able to perform complex tasks [27].

Similarly, ANNs boast similar properties but adopt a simplified process. Artificial neurons, called units, are arranged in a series of layers, independent of one another [37], and it is through these layers that data inputs are processed and returned in different forms that make sense as per the input and information sought. In the words of Huang [38], through neuromorphic devices which emulate the bio-neurons, cells, and synapses, amongst other neural components, ANNs are able to learn and train through the training set thrown at them. Graupe [28] highlighted that, just like the human brains which may be deemed simple but perform very complex duties, ANNs allow for low-level programming but are able to solve complex problems in a self-organizing manner without the need for re-programming. The artificial intelligence factor of the neural networks, highlighted by the self-programming characteristic, allows for automation of activities, leading to the wide use of ANNs $[39,40]$. This quality is deemed critical in smart cities, since one of the objectives in these cities is to have components and systems that are able to function with minimum or no human interventions.

In terms of geometry, ANNs are encoded as finite graphs or networks with direct links, in which each node represents an artificial neuron. Edges pointing to each link represent the inputs, while those pointing away from the links represent the outputs. ANNs also have hidden layers that represent the internal environment of the system. This is represented in Figure 1. 


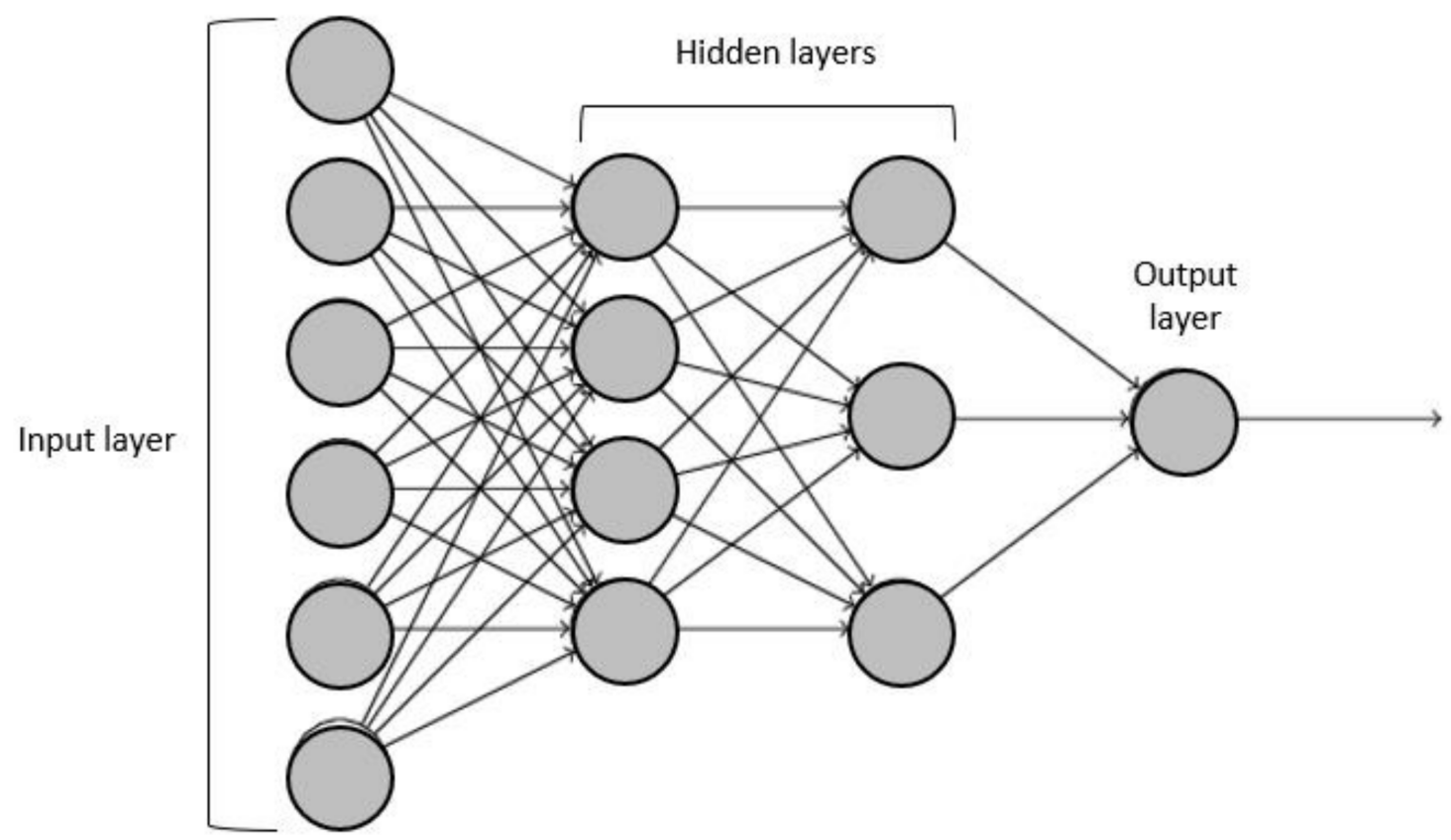

Figure 1. Geometry of a multi-layer artificial neural network (ANN).

Fukumizu [41] explained that most ANNs are multi-layered; hence, in addition to the output and input layers, they also have connections between layers in their neighborhood which are linked by weighted connections, representing the effectiveness of synaptic connections in biological neurons. To activate learning, the connection weights have to be adjusted accordingly [42].

The intricate quality of ANNs qualifies them to be used in applications related to cities which are equally intricate and complex as demonstrated by Mathaini [22] and others [21,43]. Numerous applications by other researchers were highlighted in a survey of the literature. Jafar, et al. [44] demonstrated that ANNs could be used in simulating water failures in cities, while Mathaini, Jain and Arora [21] used it to predict the expected impact of excessive land use in cities. Lee, et al. [45] made use of ANNs to predict mosquito abundances in urban areas. Using the vast applicability of ANNs, some urban fabric models such as CORINA [43], ART-MMAP [46], the ANP-ANN model for urban earthquake vulnerability assessment [47], and cellular automata (CA) [23] were developed and applied for diverse purposes.

While these models have wide-ranging applications, they were seen to be criticized as being developed in isolation, often discouraging advances that could occur from multi-disciplinary perspectives. Unlike brain neural networks that encourage complex interactions, ANNs are developed in isolation and lack the fundamental connections to enhance their processes and applicability.

\section{Brain Neural Networks and Neuroplasticity}

Human brains are reported to consist of hundreds of billions of neurons, or nerve cells, which transmit and process information [48-50]. Within each neuron, there are a hundreds of trillions of connectors called synapses that are responsible for the storage of information [32]. The neuron, which is deemed as the basic unit of the brain, consists of three structural units: the dendrites, the cell body (soma), and the axon [36]. Transmission of information is majorly undertaken by the axons and dendrites to and from the soma, where computation is deemed to take place [35]. When triggered, the impulses from the synapses are passed to the dendrites, which immediately transmit them to the soma, which in turn processes and resends the output via the axon to the synapse, and onto the next neuron until the output reaches the intended cell in the body. This is represented in Figure 2. This interconnectedness forms a complex network of neurons, through which the human brain functions. 

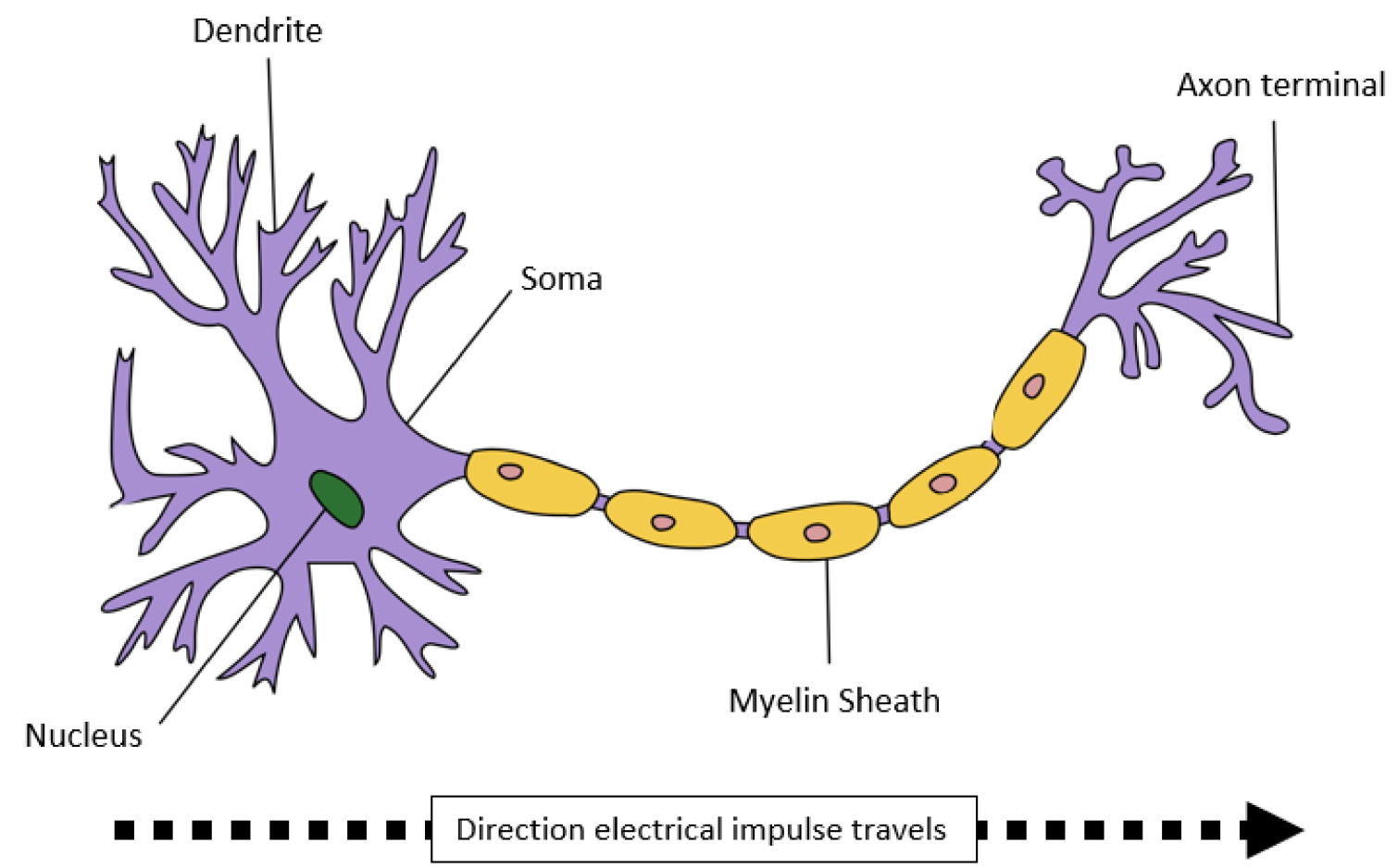

Figure 2. Communication through direct impulse in a nerve cell.

Unlike machine-stimulated neural networks which do not form new neural connections, human beings have the ability to recognize, learn, and change according to the activities in question [27] - a process called neuroplasticity or brain plasticity. Neuroplasticity can simply be defined as the brain's ability to change throughout life due to certain issues in one's experience, such as injuries and relocation, among other things. Shaffer [51] supported that this capacity of brain cells to change is influenced by both intrinsic and extrinsic factors that result from positive or negative impacts on one's life, irrespective of age. Zorins and Grabusts [27] highlighted that brain plasticity is influenced by the need for brains to grow new neurons, alter distribution and location of neurons, or alter the synaptic connections. By being able to change itself, the brain can compensate for injuries and diseases; thus, the number of neurons does not destabilize. In real life, the brain's ability to change gives hope even to individuals with medical conditions [48,49].

Human brains are susceptible to many things, and some events can result in their reprogramming, either positively or negatively, as underlined by Shaffer [50-52]. Amongst these events are the impacts of diseases and injuries on the nervous system, which are widely researched and documented. Sasmita, Kuruvilla and Ling [48] underscored that these have the potential to cause development of disorders that may affect memory, cognition, and motor functions. Bryck and Fisher [53] revealed that brain programming can also be altered by learning and training, especially in the case of children. They recognized this by examining the impact of rearing animals in an enriched environment, and the result revealed positive brain plasticity. The work of Hertzog, et al. [54] also affirmed these findings by adding that, in addition to training, other events such as physical and social activities and diverse kinds of intellectual activities have the potential to positively prompt reprogramming of the brain, especially in the elderly, for whom Mahncke, et al. [55] found out that their brains undergo changes in the form of functional losses.

In contrast to ANNs, which are trained to handle massive amounts of data and which rapidly disregard unique events, even though some are meaningful, the human brain is somehow impacted by singular important and meaningful events. This is because human brains host a complex neuronal pattern which is yet to be developed for ANNs. These patterns are influenced partly by the massive number of neurons that characterizes human brains and their interconnectedness, which provides us 
with the ability to learn different activities simultaneously, unlike ANNs which have to be trained for one particular issue at a time.

Interestingly, researchers from Carnegie Mellon [56], on an assignment to map the complex neuronal patterns of the brain on cognition and reasoning, revealed emerging maps of complex neuron geometry (Figure 3).

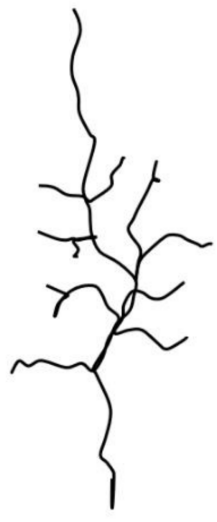

Small scale

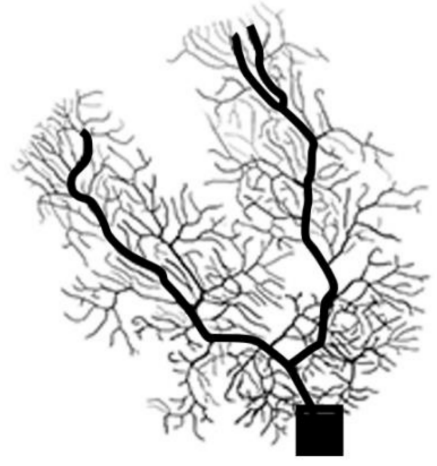

Large scale

Figure 3. Geometry of complex neuron networks.

The search for the mapping of biological, and naturally emergent, geometries was pursued by urban theorists to capitalize on the knowledge of biological entities, so as to rationalize different intricate components appertaining to cities. For instance, Salingaros [57] utilized the knowledge derived from complexity theory and pattern recognition to explain fundamental process behind urban design, thereby developing his theory of the urban web. This is seen as potentially applicable to artificial intelligence models. Alexander [58] further highlighted how urban life has to remain connected; otherwise, it precipitates to anarchy and other social problems. Other theories include the growth machine by Molotch [59], which showed that activities in cities are controlled by different social groups just like the human body is controlled by groups of neurons. The fractal theory by Batty and Longley [60] is another one that explained that elements are always different but their configuration depicts naturalness, wholeness, and uniformity just like a human body. The intricacy of networks in biological entities are, thus, seen to provide the potential to generate increased urban coherence and livability levels, which could be applied to current emerging urban concepts, such as smart cities.

\section{Emerging Networks in Smart Cities}

The Internet of things (IoT) revolutionized how cities are designed, managed, and controlled [5,7,61,62]. It is seen as the backbone of data mining in smart cities since it allows for the interconnectedness of smart devices such as autonomous vehicles, smart infrastructures, and smart sensors, among others. All these devices generate massive amounts of data, which, when analyzed, allow for real-time decision-making and responses. Kim, et al. [63] contended that, through IoT, it is now possible to monitor, manage, and remotely control all smart devices within a network. This is made possible by installing and connecting sensors ranging from RFID, IR, GPS, laser scanners, and others to the internet through unique protocols that allow seamless communication and exchange of data [64]. With these sensors in place, recognition, locating, tracking, monitoring, controlling, and management are enhanced. Data from these sensors revolutionized areas such as health, security, governance, transport, and energy sectors, amongst many others. Through data from IoT-enabled components, the use of artificial intelligence for data processing saw a rise in different environments such as business, healthcare, research and development, the financial sector, manufacturing, weather, social circles, and more $[65,66]$. 
In the recent past, there was an increase in the use of $\mathrm{AI}$ and machine learning in IoT environments. The integration of these technologies is projected to bring numerous benefits both at the device level and the larger system level. Čolaković and Hadžialić [67] contended that a combination of machine learning and AI already brought about numerous benefits ranging from data gathering to analysis and prediction, due to the applicability of ANNs in machine learning [68]. A combination of the three technologies has the potential to allow for intricate exercises such as analysis and prediction of human behavior using smart devices, for example, motion sensors and facial recognition devices [69]. These technologies also allow for smart data storage and retrieval of data and information, as well as the automation of tasks and activities in places like traffic control, street lighting, and light and temperature control in buildings, amongst others [66,70]. In the business world, Naganathan and Rao [71] explained that using these technologies will increase efficiency and productivity and provide an unprecedented competitive advantage.

A combination of IoT, AI, and machine learning will increase the quality of data gathered from IoT-enabled smart sensors, especially due to the ability of machine learning to identify patterns and errors [72]. Zhang, et al. [73] highlighted that the development and deployment of different aspects of IoT are prone to various challenges, rendering the data gathered as suspect [74,75]; however, when the data are subjected to the power of AI and machine learning, chances of bias and errors arising from the process of gathering, analyzing, and transmitting data are greatly minimized. These technologies also allow for devices to be connected to a wide range of central systems without hitches. Therefore, information on issues such as pressure, vibration, sound, pollution, and temperature will be accurate and received in real time, since the employment of ANNs in machine learning allows for quicker predictions; thus, subsequent action that needs to be taken is quick and efficient.

Looking closely at the geometry of sensors employed in smart cities, one can notice a similarity with that of biological neural networks; however, they operate in a unidimensional perspective. The information they gather as per their designing is transmitted to a central system, where it is analyzed and interpreted, and an appropriate response is sent to relevant points, where action(s) can be taken. In neural networks, in addition to input and output layers, there are hidden layers that represent the internal environment of the system, and it is here that data are analyzed, and appropriate outputs are generated. In contrast to smart cities, data gathered by smart sensors are analyzed and interpreted at the central system level. According to Spada et al. [70], this is because each network and node, serving unique sensors, was designed in silos of proprietary technologies. Conventionally, sensor networks ought to follow a universally acceptable format so as to allow for connectivity in major embedded and sensor networking operating systems [76,77]. Nevertheless, since most of the sensors are developed and programmed in isolation, they are customized to follow a homogenous network protocol; hence, the problem of inter-compatibility exists. Noting that the concept of a smart city must accommodate heterogeneity of devices and networks, the standardization of networks, as well as communication and allocation algorithms, is paramount, as expressed by Espada, et al. [78]. On the same issues, Bačić, et al. [79] proposed an interdisciplinary approach to the formulation and development of devices and protocols that are to serve the urban environment, thus fostering a good balance between actors and elements of the city. These challenges with the current forms and dimensions of ANNs showcase the need for an improved model, which is proposed below.

However, even though these technologies contribute greatly to enhancing the digital and virtual layers of smart cities, it was noted by Allam and Newman [7] that smart city technologies should primarily act as enablers and catalysts to enhance livability levels of urban fabric. To be able to support this argument, through the use of IoT, AI, and machine learning, it is important to understand how to design intricate and complex urban systems that support life. The present author guides readers toward the works of Christopher Alexander [58,80-84], which are only briefly covered in the sections below. 


\section{On Christopher Alexander's Pattern Language and Nature of Order}

\subsection{Complexity}

According to Salingaros [85], complexity, as advanced by Alexander's Pattern Language, is evoked by a score of issues. In buildings, complexity is expressed in regard to architectural language and harmony; in the eyes of the viewers, complexity is evoked by their interest in a structure, and they ascribe life to a building with respect to how complex and intricate it is perceived. The harmonious use of patterns in a building, irrespective of cost or economic qualities, is what adds to complexity. Bhat [86] supported Alexander's theory [87] that life in a building is brought about by its complexity, that is, being able to maintain a structural organization that allows for diverse elements ordered in a specific arrangement and pattern, despite there being formations and transformation on the structure. Salingaros [88] agreed with this, especially by considering how Alexander [89] emphasized the need for a living space that is comfortable and reassuring by utilizing patterns. Dawes and Ostwald [90] demonstrated this by explaining how contemporary architectures are rigid in applying formal rules and abstracts without considering how it affects the feelings of its end users. They argue that through "A Pattern Language" [87], Alexander addressed how to go about such scenarios by ensuring all the "forces" impacting on the design were addressed. He argued that life can be brought to such architectural structures via the application of different expressions and patterns. Salingaros [91] demonstrated how Alexander passionately emphasized scaling symmetry captured in fractals which are meant to make designs healthier and reassuring for users, more so since they generate biophilic patterns. Kruhl [92] supported this argument and added that complex structures should have different scaling, inhomogeneity, and anisotropy-properties dictated by fractals. Alexander believed that the use of colors, curves, and different materials renders designs complex, thus helping overcome competing forces that hinder the actualization of organized complexity [93]. It is, thus, seen that adopting and using geometrical properties can help in the design of structures to achieve complexity and its values.

\subsection{Achieving Wholeness}

In his body of works, Christopher Alexander emphasized the need for wholeness and complexity in living spaces, thus ensuring comfort and assurance to users [87,94]. These two phenomena, as Seamon [94] denoted them, constitute the basic starting point in design and structure to highlight coherence and harmony. The dimensions that the two theories emphasize are the togetherness of different parts of a structure and how they occupy their place in the whole [89]. This is demonstrated by Alexander in his work "Pattern Language" [87], by gathering examples of buildings and structures that cleverly utilize patterns to arouse harmony, complexity, and comfort. Grabow [95] affirmed that these patterns, as Alexander explained, are represented by things such as the environment and relationships of different structures, whether small or large, to form the whole. Salingaros [88] explained that the two theories also emphasize the need for life-evoking geometry in designs and structures that one is able to put in practice. In other words, it allows one to construct structures that are able to follow a step-by-step approach and flow, such that one step becomes a pointer for the next through recognition and reconciliation. By so doing, they illuminate that fractals and geometries that combine to bring about wholeness are preserved [88].

Borrowing from the concept of fractals and emerging geometries, neural networks from the brain are interestingly seen to resemble fractal scaling [96], as shown in Figure 4. 

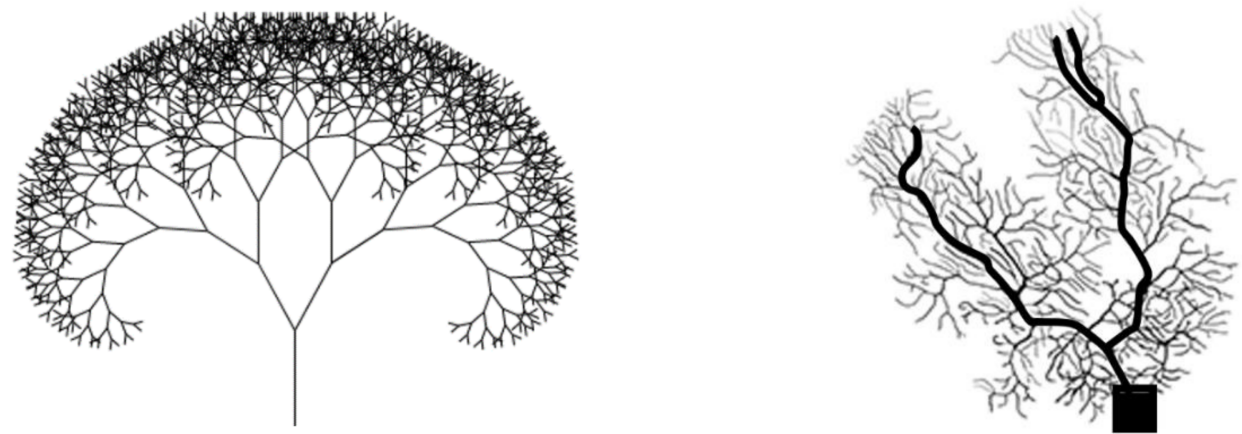

Figure 4. Geometry resemblance between fractal scaling on the left and a brain neural network on the right.

In the pursuit of wholeness, Salingaros [57] concurred with Alexander that living cities are characterized by intricate fractal properties that should not be compromised to accommodate modern trends like urbanization, population growth, or the recent trends of smart cities. Salingaros [97] explained that life in cities depends on the matrix of connectedness and substructure, and when its geometry is altered, it impacts the interaction and movement of people. He argued that, whether or not the alterations are done to integrate ICT, the intended outcomes would not be achieved unless characteristics such as fractal properties and geometry remain contact. As such, the wholeness and complexity described in "Pattern Language" are only achieved if structures remain interlinked at all scales-a quality that is compromised by urbanization, which emphasizes removing small-scale structures in favor of large-scale structures [93]. A living city is, thus, shown to need both in a balanced fashion, if it is to be deemed sustainable and livable. The current form of ANNs, which could primarily help address these challenges, was not developed in a multidimensional way; hence, despite the perceived potential of $\mathrm{AI}$ and IoT, they ultimately seem limited and require re-thinking.

\subsection{Generated Structures}

In his work, The Nature of Order [98], Alexander shared how a highly complex object is created in a process he coined as the concept of "differentiation". According to him, complexity is seen as an entity that does not arise from mere additive processes where parts are arranged and rearranged. On the contrary, he argued that building a complex structure needs to start from a whole, which is divided and differentiated into respective parts. The understanding here is that an object is not made by randomly ordering small parts in isolated fashion, but by following precise steps where the content in one step becomes the input for the next step in the whole. Salingaros [85] perceived such objects that do not follow this process as random, disordered, and unstable. Alexander [98] shared that such objects are created via the addition and arrangement of various random parts and are subject to egregious mistakes of adaptation. The adoption of the concept of differentiation, thus, provides incorporation of life in design and provides environmental sustainability outcomes while promoting well-being and wholeness [87]. Jiang [99] explained that, when the design of objects or structures abides by the proposal advanced by Alexander, they result in a kind of organic structural beauty, despite the fact that the process may be seen as a local action. He explained that such structures, which resemble complex networks, are succinctly connected between small scales and the large scale [100]. Salingaros [85] warned that random and seemingly disconnected objects invoke a feeling of boredom, depression, and, where they are large enough, oppression, since they are made from parts that add up to an object with no variation, thus rendering a monotonous object. Jiang [100] likened them to random networks and contended that they are barely connected and cannot be termed as whole.

From this, we see that structures can be made "whole" and meaningful through the interconnection of structures and networks. This somewhat differs from the current practice of unidimensional networks that ANN supports. 


\subsection{Urban Coherence}

Salingaros [101] viewed urban coherence as the relationship that exists in the city, whereby small subunits from different levels of scale, in a top-down hierarchy to the natural structure of materials, are arranged in such a way as to form a complex large-scale whole. Borrowing from Jacobs [102], she argued that this coherence is achieved when there is a high degree of organized complexity, which results in living cities and which is missing in modern cities in the way they are designed, constructed, managed, and controlled using contemporary rules. These rules, as also noted by Alexander, Ishikawa and Silverstein [87], unfortunately hamper the complexity and connectivity of moderns cities, despite the advancement in technologies and communication. Salingaros [101] underscored that urban coherence is achieved when the geometry within the city supports the whole, while maintaining flexibility, as observed in biological entities such as brain neural networks. In other words, the ability to mimic the network of paths is what defines cities, as argued by Salingaros [57]. The plasticity of the city is only possible when its fabric is well and strongly connected to the basic units that make up the city and when it is loosely connected to the large-scale units [85], as widely discussed by Alexander in his works [58,84,98]. In particular, in his book, Nature of Order, Alexander [98] explicitly explained how a complex object is created to achieve wholeness, and this description aligns with the concept of Salingaros [101] relating to urban coherence. As observed by Jiang [100], the coherence of the city results in structural beauty that is achieved by harmony and complexity of the network created by the interlinkage of different parts, differentiated from the whole, as posited by Alexander [89]. Applicable to smart cities, urban coherence is achieved when elements such as green spaces, as well as residential, commercial, pathway, and traffic infrastructures, amongst the many components, are accommodated in such a way that they foster livability and harmony despite their distinctive uniqueness. The latest technology involving ANNs is majorly unidimensional and is fronted as the platform for achieving smartness or what Alexander [98] may call whole; thus, it needs to be revisited to embrace coherence. Otherwise, smart cities will continue to operate in isolated structures through their separate and disconnected virtual and digital dimensions, thus preventing the achievement of wholeness, complexity, and the creation of life and beauty.

\section{A Proposed Theoretical Model}

A theoretical model (Figure 5) is proposed that can achieve increased intricacy and interconnectedness in smart cities through the use of ANNs. The model encourages various levels of hidden layers (denominated as dimensions 1 to $n$ ). The layers are synonymous to the various dimensions present in smart cities such as smart governance, smart environment, and smart mobility, amongst others, and each component can be coded to process information as per the criteria of that particular dimension. By ensuring that each component (and each dimension) is interconnected as depicted in the model below, a unique complexity that encourages "dialogue" of data is bound to be achieved. In particular, this is possible when the harmonization of IoT-enabled sensors and devices, especially in terms of protocols and standards, is emphasized. This way, multidimensional processes and interactions are made possible. As current ANN processing is restricted in the type of data processing through the selected data computed, there is limited room for engagement and cross database reading; thus, it reduces the opportunities for further computing during the same process. This model is different as it promotes the need for a uniform system and network, which serves as a series of interconnected devices, sensors, and systems, particularly available in smart cities. This will ensure that the processing engages a variety of gathered data from various segments from the standardized IoT network.

Figure 5 demonstrates how data borne from various components of the city can be linked to one single ANN system, such that the data generated and analyzed are made to be integrated cohesively; hence, the output response that will be relayed in different dimensions of the city, depicted by components $1 \mathrm{~A}$ to $\mathrm{nE}$, will be reliable and quality, and the action taken is expected to be the most potent. 

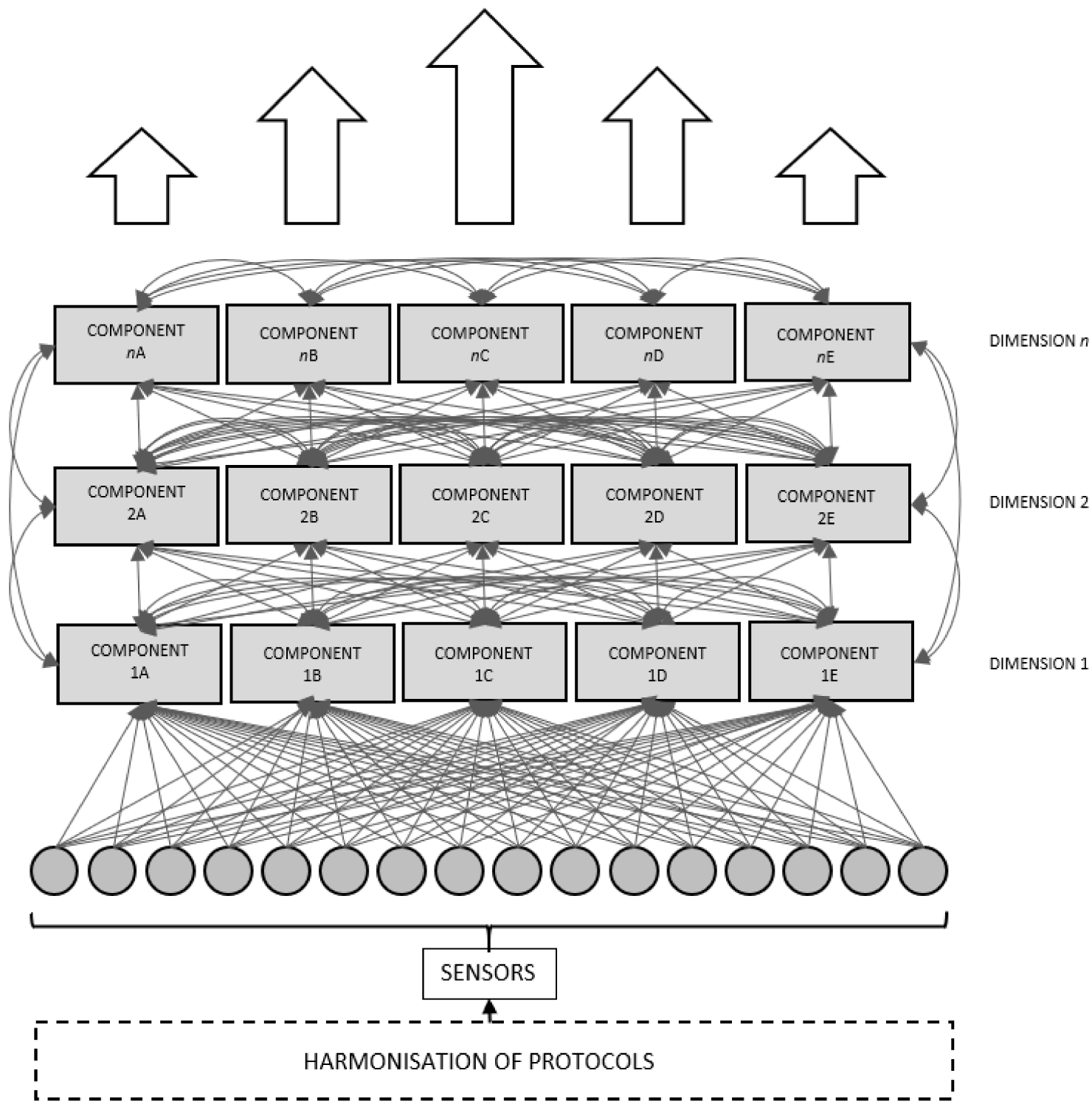

Figure 5. Theoretical model for achieving increased complexity in smart cities.

It was demonstrated in the literature above that existing models suffer from a myriad of challenges brought about by the homogeneity of protocols and systems relied upon by the smart city components. Adoption of this proposed model will ensure that all the components of the city are interlinked to one another, just like the brain's neurons are; hence, there will be no need for maintaining multiple systems while ensuring increased data collection and processing. This allows for one central server, from which data from every city component can be connected, analyzed, and dispatched to respective output nodes.

\section{Discussion}

The actualization of the concept of smart cities toward an intelligent, resilient, and complex system is attainable. As showcased from the literature above, the available technologies such as IoT, AI, and machine learning have the potential to spur this reality $[63,64,67]$. Nevertheless, there ought to be a collaborative and interdisciplinary approach to avoid the pitfalls extensively discussed by Alexander in his book "Pattern Language" [87] and his extensive volumes "The Order of Order" [98]. He pointed out the concept of rigidity and a unidimensional approach, as confirmed by other authors [73-75], which affects the current technologies employed to enable the implementation of smart cities portrayed 
by current ANN networks and structures. In an ideal situation, Alexander advised that structures or designs should be viewed in terms of their whole, developing from that point downward. Contrary to this, the current approach of implementing smart cities by randomly assembling smart parts, with the aim of forming a concrete whole, is not a sustainable and intelligent undertaking. The proposed model addresses the shortcomings of current ANN networks and structures by emphasizing the need to have standardized and uniform protocols that are compatible with smart components regardless of their manufacturer. Through the adoption of this concept, parameters such as costs of securing different components would be minimized. Similarly, this will provide an opportunity to equally address issues relating to a lack of trust that bedevils the smart city concept due to the perceived intrusion of personal security.

It was established that, though different parties including scientists, planners, urbanists, and urban leaders, where there is increased interest to develop designs and structures that would encourage sustainability and foster livability, there is a notable lack of collaboration [79]. Indeed, it was revealed that, starting from the basic premise that Alexander [98] referred to as the small scale, different groups are working in isolation. For instance, it was established that the different smart devices and networks in smart cities that are relied upon in gathering vital data do not run from a harmonized protocol, but each rely on a homogenous technology dictated by the manufacturers $[76,78]$. For this reason, multiple networks and numerous central nervous systems are maintained-a practice that compromises the quality, quantity, and accuracy of data received. Technology standardization is lacking in areas like communication, data aggregation, and networks, and these challenges affect the optimization of IoT capabilities. Without standardization, the simplicity of the system that Alexander advocated for cannot be attained. Nevertheless, adopting the proposal in the model presented above can help reverse the current situation, as collaboration at every level of development of the smart city is emphasized.

In particular, one area that requires collaboration is the development of ANNs, which was left in the hands of scientists who have little knowledge of how the different components of the urban fabric affect issues like livability, sustainability, and economic growth. Like the biological neurons from which developers of ANNs derive their motivation, the development of programs requires input from all stakeholders, especially the designers, planners, and managers of the city. The human brain is said to receive impulses from different cells in the body, and, for this reason, when required, they undergo neuroplasticity to accommodate or adhere to the situation. Likewise, ANNs, although not as plastic as the brain, ought to be developed in a complex and stronger way as proposed in the model presented above, such that they can process data in a multidimensional way rather than the traditional unidimensional form. From the literature, different proponents of Alexander's works $[85,95,99]$ advanced the idea of having more connections in the city to allow for harmony, wellness, and livability. Likewise, development and training of ANNs need to accommodate the inputs of different stakeholders in smart city implementation. The development of ANNs should be guided by firstly understanding the design of the proposed smart city so that even the smaller components are factored in the final fashion of ANNs dedicated for smart cities. That way, components such as the sensors, smart devices, buildings, streets, networks, and the central nervous system can be wired in a uniform platform; hence, the concept of increased efficiency and performance that characterize smart cities is achieved. While this may be seen as a challenge, the advantages are seen to provide increased efficiency and coherence, and they can help in addressing parallel issues of privacy and communication concerns [(Allam, 2019 \#677)]. However, this may face some resistance from an economic perspective, as ICT corporations would be expected to favor specific networks riding on proprietary technology, thus ensuring market monopoly. Although this is the case, there have numerous calls from various authors [5,7,103-105] to highlight the economic benefits for economic inclusivity through the democratization of smart city technologies and ICT networks, thereby leading to a wider adoption and resulting in higher profit margins by ICT corporations. 
By ensuring that different stakeholders are involved during the development of ANNs, as proposed in the theoretical model advanced in this paper, the training set would be robust, and, as Zorins and Grabusts [27] highlighted, this would allow it to respond to a wide range of new challenges characterized by cities.

The ideas advanced by Alexander, relating to designs and structures that seek to arouse liveliness in users [89] by applying the use of patterns, were identified to influence contemporary thinking, such as in the emerging concept of smart cities. The wealth of works by Alexander provides a blueprint to achieve complexity and wholeness [101], and it can be made applicable to machine learning and can provide the emergence of economic gains across emerging fields. It is seen that from literature, especially on modern technologies, that this is achievable [76]. In smart cities, complexity and wholeness are represented by the ability of the city to accommodate sustainability, green spaces, and optimal use of resources, having residential areas that foster equality and social inclusion and promote economic growth. As complexity is achieved by the interconnectedness and simplicity from the combination of small-scale parts to form beautiful large-scale entities, as referred by Jiang [100], the concept of smart cities can be optimized by improving the basic elements that would add up to a complete whole. Such basic elements include the strengthening of the networks and standardizing the operating protocols, especially those related to IoT, for an increased machine learning potential.

\section{Conclusions}

This paper explored, through a multi-disciplinary perspective, how to transcend the limitations of artificial neural networks to achieve neuroplasticity through smart cities by increasing the complexity of its geometrical entities. Christopher Alexander's Nature of Order, as well as his theory of complexity and wholeness, was used as the underlying foundation to understand how to achieve this level of intricacy while keeping the richness of communication in networks and systems. The principles led to the proposed model that makes use of data from IoT's sensors and manages them through a new ANN framework. Through this, the principle of neuroplasticity is seen to be achievable, majorly because the proposed model envisions a more intricate and complex system that has the ability to accommodate more learning sets. Through this line, it was noted that IoT devices in smart cities need to be harmonized in terms of protocols and standards; adoption of the proposed model offers the opportunity for this to happen. It is further understood that the dimension of collaboration between different stakeholders and smart city suppliers is paramount. This is critical, as, in order to facilitate and accelerate the adoption of the Smart City concept, a platform, such as the one proposed in this paper, needs to be built where collective data can provide the identification of emerging patterns and synergies, thus leading to an increase in efficiency and performance of urban management. This will provide an increased potential to use data across various platforms and to render more intricate and complex processing to render more sustainable, resilient, safe, and vibrant urban environments.

Funding: No funding was received for this study.

Conflicts of Interest: The author declare no conflict of interest.

\section{References}

1. OECD. Cities and climate change: National governments enabling local action. In OECD Policy Perspectives; Organisation for Economic Co-operation and Development; OECD: Paris, France, 2014; pp. 1-21.

2. United Nations. Sustainable Development Goals Report; UN: New York, NY, USA, 2018; p. 40.

3. UNDP. Sustainable Urbanization Strategy: Undp's Support to Sustainable, Inclusive and Resilient Cites in the Developing World; United Nations Development Programme: New York, NY, USA, 2016.

4. Sepasgozar, S.M.E.; Hawken, S.; Sargolzaei, S.; Foroozanfa, M. Implementing citizen centric technology in developing smart cities: A model for predicting the acceptance of urban technologies. Technol. Forecast. Soc. Chang. 2018, 142, 115-116. [CrossRef] 
5. Allam, M.Z. Redefining the Smart City: Culture, Metabolism and Governance. Case Study of Port Louis, Mauritius; Curtin University: Perth, Australia, 2018.

6. Allam, Z. Contextualising the smart city for sustainability and inclusivity. New Des. Ideas 2018, 2, $124-127$.

7. Allam, Z.; Newman, P. Redefining the smart city: Culture, metabolism \& governance. Smart Cities 2018, 1, $4-25$.

8. Barns, S.; Cosgrave, E.; Acuto, M.; Mcneill, D. Digital infrastructures and urban governance. Urban Policy Res. 2016, 35, 20-31. [CrossRef]

9. Gil, D.; Ferrández, A.; Moramora, H.; Peral, J. Internet of things: A review of surveys based on context aware intelligent services. Sensors 2016, 16, 1069. [CrossRef]

10. Bibri, S.E. The ioT for smart sustainable cities of the future: An analytical framework for sensor-based big data applications for environmental sustainability. Sustain. Cities Soc. 2018, 38, 230-253. [CrossRef]

11. Bassoo, V.; Ramnarain-Seetohul, V.; Hurbungs, V.; Fowdur, T.P.; Beeharry, Y. Big data analytics for smart cities. In Internet of Things and Big Data Analytics toward Next-Generation Intelligence. Studies in Big Data; Dey, N., Hassanien, A., Bhatt, C., Stapathy, S., Eds.; Springer: Cham, Switzerland, 2018; Volume 30.

12. Batty, M. Big data and the city. Built Environ. 2016, 42, 321-337. [CrossRef]

13. Al-Saqaf, W.; Seidler, N. Blockchain technology for social impact: Opportunities and challenges ahead. J. Cyber Policy 2017, 2, 338-354. [CrossRef]

14. Christidis, K.; Devetsikiotis, M. Blockchains and smart contracts for the internet of things. IEEE Access 2016, 4, 2292-2303. [CrossRef]

15. Allam, Z. On smart contracts and organisational performance: A review of smart contracts through the blockchain technology. Rev. Econ. Bus. Stud. 2018, 11, 137-156. [CrossRef]

16. Bini, S.A. Artificial intelligence, machine learning, deep learning, and cognitive computing: What do these terms mean and how will they impact health care? J. Arthroplast. 2018, 33, 2358-2361. [CrossRef] [PubMed]

17. Guo, K.; Lu, Y.; Gao, H.; Cao, R. Artificial intelligence-based semantic internet of things in a user-centric smart city. Sensors 2018, 18, 1341. [CrossRef]

18. Aguilera, U.; Peña, O.; Belmonte, O.; López-de-Ipiña, D. Citizen-centric data services for smarter cities. Future Gener. Comput. Syst. 2017, 76, 234-247. [CrossRef]

19. Khan, M.; Babar, M.; Ahmed, S.H.; Shad, S.C.; Han, K. Smart city designing and planning based on big data analytics. Sustain. Cities Soc. 2017, 35, 271-279. [CrossRef]

20. Silva, B.N.; Khan, M.; Han, K. Towards sustainable smart cities: A review of trends, architectures, components, and open challenges in smart cities. Sustain. Cities Soc. 2018, 38, 697-713. [CrossRef]

21. Mathaini, S.; Jain, R.K.; Arora, M.K. An artificial neural network based approach for modelling urban spatial growth. ITPI J. 2007, 4, 43-51.

22. Mathaini, S. A neural network based urban growth model of an Indian city. J. Indian Soc. Remote Sens. 2009, 37, 363-376. [CrossRef]

23. Yeh, A.G.; Li, X. Urban simulation using neural networks and cellular automata for land use planning. In Proceedings of the Symposium on Geospatial Theory, Processing and Applications, Ottawa, ON, Canada, 9-12 July 2002.

24. Sorda, K.R. Artificial Neural Networks-The Future of Smart Cities. Available online: http://www. itwebafrica.com/home-pagex/opinion/244318-artificial-neural-networks--the-future-of-smart-cities (accessed on 1 January 2019).

25. Dubey, A.; Naik, N.; Parikh, D.; Raskar, R.; Hidalgo, C.A. Deep learning the city: Quantifying urban perception at a global scale. In Proceedings of the ECCV European Conference on Computer Vision, Amsterdam, The Netherlands, 11-14 October 2016.

26. Kropp, J. A neural network approach to the analysis of city systems. Appl. Geogr. 1998, 18, 83-96. [CrossRef]

27. Zorins, A.; Grabusts, P. Artificial Neural Networks and Human Brain: Survey of Improvement Possibilities of Learning. In Proceedings of the 10th International Scientific and Practical Conference, Rezekne, Latvia, 18-20 June 2015; pp. 228-231.

28. Graupe, D. Principles of Artificial Neural Networks: Advanced Series on Circuits and Systems, 2nd ed.; World Scientific Publishing Co. Ltd.: Singapore, 2007; Volume 6.

29. Jain, L.; Fanelli, A.M. Recent Advances in Artificial Neural Networks: Design and Applications; CRC Press LLC: Boca Raton, FL, USA, 2000. 
30. Mehrotra, K.; Mohan, C.K.; Ranka, S. Elements of Artificial Neural Networks; MIT Press: Cambridge, MA, USA, 1997.

31. Botta, A.; Donato, W.D.; Persico, V.; Pescapé, A. Integration of cloud computing and internet of things: A survey. Future Gener. Comput. Syst. 2016, 56, 684-700. [CrossRef]

32. Solomon, A.S.; Henri, C.; Charles, S.; Melwin, P.; Ninoshka, T. Neuron the memory unit of the brain. IOSR J. Comput. Eng. 2015, 17, 48-61.

33. Haykin, S. Neural Networks and Learning Machines, 3rd ed.; McMaster University Hamilton: Ontario, ON, Canada, 2009.

34. He, H.; Garcia, E.A. Learning from imbalanced data. IEEE Trans. Knowl. Data Eng. 2009, 21, 1260-1284.

35. Sidiropulou, K.; Pissadaki, E.K.; Poirazi, P. Inside the brain of a neuron. EMBO Rep. 2006, 7, 886-892. [CrossRef]

36. Perwej, Y.; Parwej, F. A neuroplasticity (brain plasticity) approach to use in artificial neural network. Int. J. Sci. Eng. Res. 2012, 3, 1-9.

37. Abiodun, O.I.; Jantan, A.; Omolara, A.E.; Dada, K.V.; Mahamed, N.A.; Arshad, H. State-of-the-art in artificial neural network applications: A survey. Heliyon 2018, 4, e00938-e00979. [CrossRef] [PubMed]

38. Huang, T.-J. Imitating the brain with neurocomputer: A "new" way towards artificial general intelligence. Int. J. Autom. Comput. 2017, 14, 520-531. [CrossRef]

39. GK. How Neural Networks Work. Available online: https:// chatbotslife.com/how-neural-networks-workff4c7ad371f7 (accessed on 2 January 2019).

40. Kurama, V. Deep Learning with Python. Available online: https://towardsdatascience.com/deep-learningwith-python-703e26853820 (accessed on 3 January 2019).

41. Fukumizu, K. Chapter 17-Geometry of neural networks: Natural gradient for learning. In Handbook of Biological Physics; Moss, F., Gielen, S., Eds.; Elsevier: Amsterdam, The Netherlands, 2001; pp. 731-769.

42. Hauser, M.; Ray, A. Principles of Riemannian geometry in neural networks. In Proceedings of the 31st Conference on Neural Information Processing Systems NIPS, Long Beach, CA, USA, 4-9 December 2017.

43. Triantakonstantis, D.; Stathakis, D. Urban growth prediction in Athens, Greece, using artificial neural networks. Int. J. Archit. Environ. Eng. 2015, 9, 234-238.

44. Jafar, R.; Shahrour, I.; Juran, I. Application of artificial neural networks (ANN) to model the failure of urban water mains. Math. Comput. Model. 2010, 51, 1170-1180. [CrossRef]

45. Lee, K.Y.; Chung, N.; Hwang, S. Application of an artificial neural network (ANN) model for predicting mosquito abundances in urban areas. Ecol. Inform. 2016, 36, 172-180. [CrossRef]

46. Liu, W.; Seto, K.C. Using the ART-MMAP neural network to model and predict urban growth: A spatio-temporal data mining approach. Environ. Plan. B Plan. Des. 2008, 35, 296-317. [CrossRef]

47. Alizadeh, M.; Ngah, I.; Hashim, M.; Pradhan, B.; Pour, A.B. A hybrid analytic network process and artificial neural network (ANP-ANN) model for urban earthquake vulnerability assessment. Remote Sens. 2018, 10, 975. [CrossRef]

48. Sasmita, A.O.; Kuruvilla, J.; Ling, A.P.K. Harnessing neuroplasticity: Modern approaches and clinical future. Int. J. Neurosci. 2018, 128, 1061-1077. [CrossRef]

49. Aleksandra, C. How Is a Neuron Adapted to Perform Its Function. Available online: https:/ / socratic.org/ questions/how-is-a-neuron-adapted-to-perform-its-function (accessed on 3 January 2019).

50. Pascual-Leone, A.; Amedi, A.; Fregni, F.; Merabet, L.B. The plastic human brain cortex. Annual Review of Neuroscience 2005, 28, 377-401. [CrossRef]

51. Shaffer, J. Neuroplasticity and clinical practice: Building brain power for health. Front. Psychol. 2016, 7, 1118. [CrossRef]

52. Schaefer, N.; Rotermund, C.; Blumrich, E.-M.; Lourenco, M.V.; Joshi, P.; Hegemann, R.U.; Jamwal, S.; Ali, N.; Romero, E.M.G. The malleable brain: Plasticity of neural circuits and behavior-A review from students to students. J. Neurochem. 2017, 142, 790-811. [CrossRef]

53. Bryck, R.L.; Fisher, P.A. Training the brain: Practical applications of neural plasticity from the intersection of cognitive neuroscience, developmental psychology, and prevention science. Am. Psychol. 2012, 67, 87-100. [CrossRef]

54. Hertzog, C.; Kramer, A.F.; Wilson, R.S.; Lindenberger, U. Enrichment effects on adult cognitive development: Can the functional capacity of older adults be preserved and enhanced? Psychol. Sci. Public Interest J. Am. Psychol. Soc. 2008, 9, 1-65. [CrossRef] 
55. Mahncke, H.W.; Bronstone, A.; Merzenich, M.M. Brain plasticity and functional losses in the aged: Scientific bases for a novel intervention. Prog. Brain Res. 2006, 157, 81-109.

56. Yang, M. Mapping the Brain's Traffic. Available online: https://engineering.cmu.edu/news-events/news/ 2018/09/06-mapping-brain-traffic.html (accessed on 3 January 2019).

57. Salingaros, N.A. Theory of the urban web. J. Urban Des. 1998, 3, 53-71. [CrossRef]

58. Alexander, C. A city is not a tree. Archit. Forum 1965, 122, 58-61.

59. Molotch, H. The city as a growth machine: Toward a political economy of place. Am. J. Sociol. 1976, 82, 309-332. [CrossRef]

60. Batty, M.; Longley, P. Fractal Cities: A Geometry of Form and Function; Academic Press: Cambridge, MA, USA, 1994.

61. Allam, Z. Building a conceptual framework for smarting an existing city in Mauritius: The case of Port Louis. J. Biourbanism 2017, 4, 103-121.

62. Allam, Z.; Newman, P. Economically incentivising smart urban regeneration. Case study of Port Louis, Mauritius. Smart Cities 2018, 1, 53-74. [CrossRef]

63. Kim, T.-h.; Ramos, C.; Mohammed, S. Smart city and IoT. Future Gener. Comput. Syst. 2017, 76, $159-162$. [CrossRef]

64. Qin, Y.; Sheng, Q.Z.; Falkner, N.J.; Dustdar, S.; Wang, H.; Vasilakos, A.V. When things matter: A survey on data-centric internet of things. J. Netw. Comput. Appl. 2016, 64, 137-153. [CrossRef]

65. Deshpande, A.; Pitale, P.; Sanap, S. Industrial automation using internet of things (IoT). Int. J. Adv. Res. Comput. Eng. Technol. (IJARCET) 2016, 5, 266-269.

66. Tzafestas, S.G. Synergy of IoT and AI in modern society: The robotics and automation case. Robot. Autom. Eng. J. 2018, 31, 1-15. [CrossRef]

67. Čolaković, A.; Hadžialić, M. Internet of things (IoT): A review of enabling technologies, challenges, and open research issues. Comput. Netw. 2018, 144, 17-39. [CrossRef]

68. Kotenko, I.; Saenko, I.; Skorik, F.; Bushuev, S. Neural network approach to forecast the state of the internet of things elements. In Proceedings of the XVIII International Conference on Soft Computing and Measurements (SCM), St. Petersburg, Russia, 19-21 May 2015; pp. 133-135.

69. Mahdavinejad, M.S.; Rezvan, M.; Barekatain, M.; Adabi, P.; Barnaghi, P.; Sheth, A.P. Machine learning for internet of things data analysis: A survey. Digit. Commun. Netw. 2018, 4, 161-175. [CrossRef]

70. Forsyth, R. Expert Systems; Chapman and Hall: Boca Raton, FL, USA, 2017.

71. Naganathan, V.; Rao, R.K. The evolution of internet of things: Bringing the power of artificial intelligence to iot, its opportunities and challenges. Int. J. Comput. Sci. Trends ADN Technol. 2018, 6, 94-108.

72. Patel, K.K.; Patel, S.M. Internet of things-IoT: Definition, characteristics, architecture, enabling technologies, applications and future challenges. Int. J. Eng. Sci. Comput. 2016, 6, 6122-6131.

73. Zhang, N.; Chen, J.; Chen, X.; Chen, J. Semantic framework of internet of things for smart cities: Case studies. Sensors 2016, 16, 1501. [CrossRef]

74. Barnaghi, P.; Bermudez-Edo, M.; Tonjes, R. Challenges for quality of data in smart cities. J. Data Inf. Qual. 2015, 6, 6. [CrossRef]

75. Keenan, M. The future of data with the rise of the IoT. RFID J. 2018. Available online: https://www. rfidjournal.com/articles/view?17954 (accessed on 25 January 2019).

76. Khaled, A.E.; Halal, S. Interoperable communication framework for bridging restful and topic-Based communication in IoT. Future Gener. Comput. Syst. 2019, 92, 628-643. [CrossRef]

77. Al-Fugaha, A.; Guizani, A.M.; Mehdi, M.; Mohammed, A.; Moussa, A. Internet of things: A survey on enabling technologies, protocols, and applications. IEEE Commun. Surv. Tutor. 2015, 17, 2347-2376. [CrossRef]

78. Espada, J.P.; Yager, R.; Yu, Z. Communications, collaborations and services in networks of embedded devices. Future Gener. Comput. Syst. 2019, 92, 560-563. [CrossRef]

79. Bačić, Ž.; Jogun, T.; Majić, I. Integrated sensor systems for smart cities. Teh. Vjesn. 2018, 25, 277-284.

80. Alexander, C. A New Theory of Urban Design; Oxford University Press: New York, NY, USA, 1987.

81. Alexander, C. The Process of Creating Life: An Essay on the Art of Building and the Nature of the Universe; Center for Environmental Structure: Berkeley, CA, USA, 2002.

82. Alexander, C. The Phenomenon of Life: An Essay on the Art of Building and the Nature of the Universe; Center for Environmental Structure: Berkeley, CA, USA, 2002. 
83. Alexander, C. The Luminous Ground: An Essay on the Art of Building and the Nature of the Universe; Center for Environmental Structure: Berkeley, CA, USA, 2002.

84. Alexander, C. A Vision of a Living World: An Essay on the Art of Building and the Nature of the Universe; Center for Environmental Structure: Berkeley, CA, USA, 2002.

85. Salingaros, N.A. Complexity in architecture and design. Oz J. 2014, 36, 18-25. [CrossRef]

86. Bhat, R. Understanding complexity through pattern languages in biological and man-made architectures. Archnet Int. J. Archit. Res. 2014, 8, 8-19. [CrossRef]

87. Alexander, C.; Ishikawa, S.; Silverstein, M. A Pattern Language; Oxford University Press: New York, NY, USA, 1977.

88. Salingaros, N.A. Connecting the fractal city. In 5th Biennial of Towns and Town Planners in Europe; PLANUM: Barcelona, Spain, 2003; pp. 78-101.

89. Alexander, C. The Timeless Way of Building; Oxford University Press: New York, NY, USA, 1979.

90. Dawes, M.J.; Ostwald, M.J. Christopher Alexander's a pattern language: Analysing, mapping and classifying the critical response. City Territ. Archit. 2017, 4, 1-14. [CrossRef]

91. Salingaros, N.A. Biophilia and Healing Environments; Off the Common Books: Amherst, MA, USA, 2015.

92. Kruhl, J.H. Fractal-geometry techniques in the quantification of complex rock structures: A special view on scaling regimes, inhomogeneity and anisotropy. J. Struct. Geol. 2013, 46, 2-21. [CrossRef]

93. Salingaros, N.A. Fractals and Christopher Alexander's "fifteen fundamental properties". In Conscious Cities Anthology 2018; Human-Centred Design: London, UK, 2018.

94. Seamon, D. Christopher Alexander and a phenomenology of wholeness. In Christopher Alexander Annual Meeting of the Environmental Design Research Association; EDRA: Sacramento, CA, USA, 2007.

95. Grabow, S. Christopher Alexander and the Search for a New Paradigm in Architecture; Oriel Press: London, UK, 1983.

96. Delaney, S. Fractal Scaling. Available online: https://medium.com/@sdelaney32/fractal-scaling-6dc8fa1773a9 (accessed on 3 January 2019).

97. Salingaros, N.A.; Fractals in the new architecture. Archimagazine 2001. Available online: http:/ / zeta.math. utsa.edu/ \{\}yxk833/fractals.html (accessed on 25 January 2019).

98. Alexander, C. The Nature of Order: The Process of Creating Life; The Centre for Environmental Structure: Berkeley, CA, USA, 2002.

99. Jiang, B. A new kind of beauty out of the underlying scaling of geographic space. Prof. Geogr. 2014, 66, 676-686. [CrossRef]

100. Jiang, B. Wholeness as a hierarchical graph to capture the nature of space. Int. J. Geogr. Inf. Sci. 2015, 29, 1632-1648. [CrossRef]

101. Salingaros, N.A. Complexity and urban coherence. J. Urban Des. 2000, 5, 291-316. [CrossRef]

102. Jacobs, J. The Death and Life of Great American Cities; Random House: New York, NY, USA, 1961.

103. Allam, Z.; Dhunny, A.; Siew, G.; Jones, D. Towards smart urban regeneration: Findings of an urban footprint survey in Port Louis, Mauritius. Smart Cities 2018, 1, 121-133. [CrossRef]

104. Allam, Z.; Dhunny, Z.A. On big data, artificial intelligence and smart cities. Cities 2019, 89, 80-91. [CrossRef]

105. Allam, Z.; Jones, D.S. The potential of blockchain within air rights development as a prevention measure against urban sprawl. Urban Science 2019, 3, 38. [CrossRef]

(C) 2019 by the author. Licensee MDPI, Basel, Switzerland. This article is an open access article distributed under the terms and conditions of the Creative Commons Attribution (CC BY) license (http:/ / creativecommons.org/licenses/by/4.0/). 